PAPER Special Section on Cognitive Radio and Spectrum Sharing Technology

\title{
Asynchronous, Decentralized DS-CDMA Using Feedback-Controlled Spreading Sequences for Time-Dispersive Channels
}

\author{
Teruhiko MIYATAKE ${ }^{\dagger}$, Nonmember, Kazuki CHIBA ${ }^{\dagger \dagger}$, Student Member, Masanori HAMAMURA ${ }^{\dagger a)}$, \\ and Shin'ichi TACHIKAWA ${ }^{\dagger \dagger \dagger}$, Members
}

\begin{abstract}
SUMMARY We propose a novel asynchronous direct-sequence codedivision multiple access (DS-CDMA) using feedback-controlled spreading sequences (FCSSs) (FCSS/DS-CDMA). At the receiver of FCSS/DSCDMA, the code-orthogonalizing filter (COF) produces a spreading sequence, and the receiver returns the spreading sequence to the transmitter. Then the transmitter uses the spreading sequence as its updated version. The performance of FCSS/DS-CDMA is evaluated over time-dispersive channels. The results indicate that FCSS/DS-CDMA greatly suppresses both the intersymbol interference (ISI) and multiple access interference (MAI) over time-invariant channels. FCSS/DS-CDMA is applicable to the decentralized multiple access.

key words: spectrum sharing, spread spectrum, spreading sequence, direct sequence, multipath, COF, RAKE
\end{abstract}

\section{Introduction}

In general, the performance of digital communication systems is deteriorated by intersymbol interference (ISI) due to multipath and multiple access interference (MAI) [1][4]. Even though ISI and MAI exist over a transmission channel, direct-sequence code-division multiple access (DSCDMA) can suppress them by means of the processing gain (or by the process of despreading in the time domain at the receiver) and can improve the signal-to-interference-andnoise ratio (SINR). In addition, the DS-CDMA can further improve the SINR by applying a combining method such as RAKE and Pre-RAKE [1], [4], [5]. However, the suppression capability for ISI and MAI declines in DS-CDMA when the processing gain is not sufficiently large to achieve high-speed data communications within a given bandwidth.

Another approach for ISI and MAI suppression (or cancellation) in DS-CDMA is to apply adaptive filters [6]-[9]. Adaptive filters can reduce ISI and MAI without any explicit knowledge of the channels. However, the signal-to-noise ratio (SNR) declines when the ISI and MAI components are correlated to the desired component of the received signal. In order to suppress MAI, orthogonal analog spreading se-

\footnotetext{
Manuscript received May 7, 2007.

Manuscript revised July 24, 2007.

${ }^{\dagger}$ The authors are with the Graduate School of Engineering, Kochi University of Technology, Kami-shi, 782-8502 Japan.

${ }^{\dagger}$ The author is with the Department of Engineering, Kochi University of Technology, Kami-shi, 782-8502 Japan.

${ }^{\dagger \dagger \dagger}$ The author is with the Department of Electrical Engineering, Nagaoka University of Technology, Nagaoka-shi, 940-2188 Japan.

a) E-mail: hamamura.masanori@kochi-tech.ac.jp

DOI: $10.1093 /$ ietcom/e91-b.1.53
}

quences produced by adaptive filter were proposed for reverse link DS-CDMA [9]. Since the analog spreading sequences require a base station (BS) for the orthogonalization, it cannot be directly applied to decentralized multiple access where no BS exists.

In this paper, we propose a novel DS-CDMA (FCSS/ DS-CDMA) that employs feedback-controlled spreading sequences (FCSS) [10]-[12] to suppress ISI and MAI, which results in efficient bandwidth sharing for decentralized multiple access over asynchronous and time-invariant (or almost time-invariant) channels. The receiver for each user is composed of a code-orthogonalizing filter (COF) [7] and only sends back information about the converged weights of the COF to the transmitter several times.

In the following sections, first, we describe the system model and the adaptive algorithm used at the receiver. Second, the bit-error rate (BER) performance of the FCSS/DSCDMA is evaluated in comparison with the conventional DS-CDMA with/without RAKE combining over two-path and powerline channels. The influence of quantizing error on the FCSS is also investigated in terms of the BER in Sect. 3. Finally, our results are summarized in Sect. 4.

\section{System Model}

\subsection{Transmitter}

We assume an asynchronous, decentralized DS-CDMA where $U$ simultaneous users are accommodated. Figure 1 shows the DS-CDMA, and the transmitter $\mathrm{T}_{\mathrm{x}, u}$ and receiver

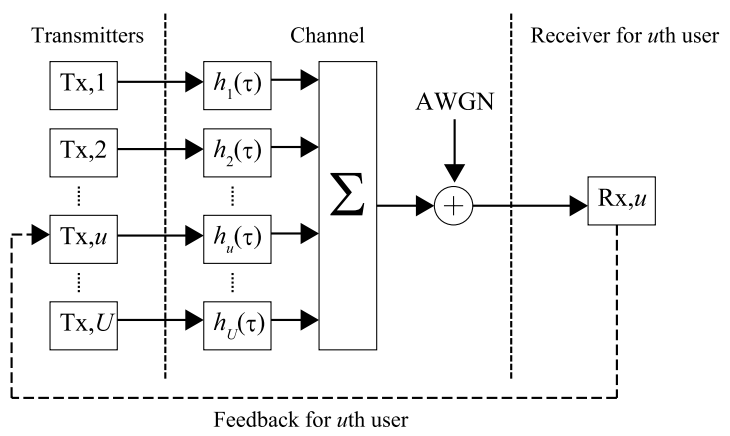

Fig. 1 Asynchronous, decentralized DS-CDMA. 


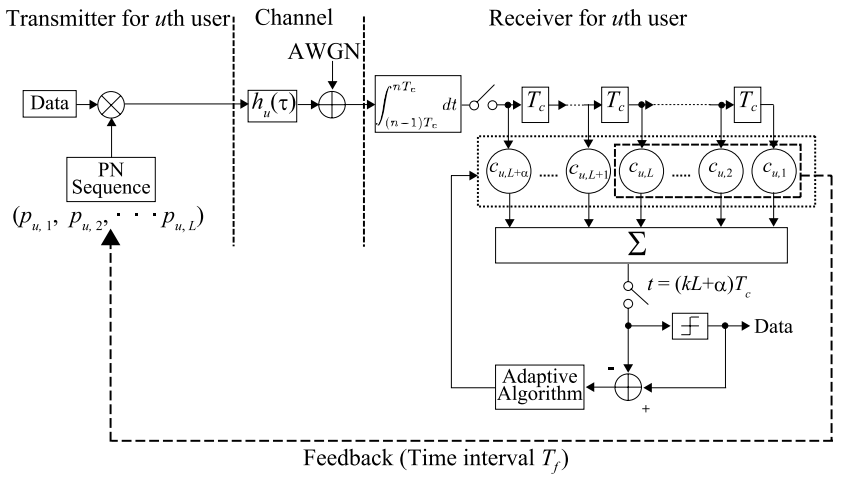

Fig. 2 Transmitter and receiver for $u$ th user.

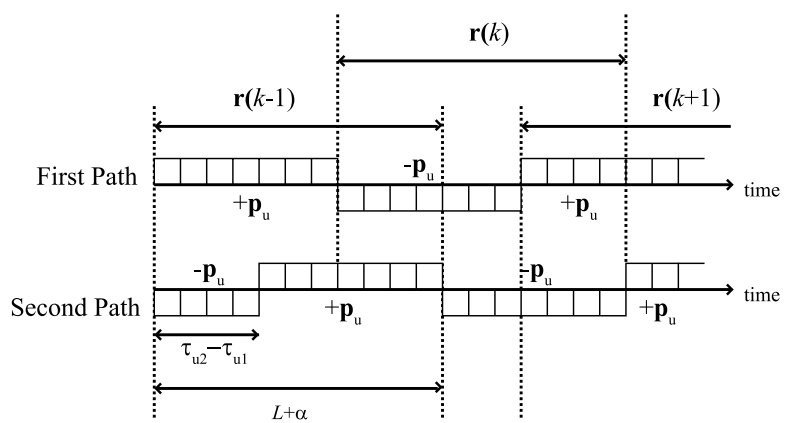

Fig. 3 Received signals through a two-path channel with relative delay $\tau_{u}\left(=\tau_{u 2}-\tau_{u 1}\right)=4 T_{c}$ for $u$ th user $(L=7$ and $\alpha=4)$.

$\mathrm{R}_{\mathrm{x}, u}$ for the $u$ th user $(u=1,2, \ldots, U)$ are shown in Fig. 2 . In Figs. 1 and $2, h_{u}(\tau)$ denotes the impulse response of the channel for the $u$ th user.

Suppose that data signals are in a bipolar NRZ (nonreturn-to-zero) format and that the spreading sequence of length $L$ for the $u$ th user is $\left(p_{u, 1}, p_{u, 2}, \ldots, p_{u, L}\right)$, as shown in Fig. 2. The signals of the data and the chips of the spreading sequence are assumed to be rectangular pulses and their durations are $T_{s}$ and $T_{c}$ [s], respectively. We assume that $T_{s}=L T_{c}$. The data signal multiplied by the spreading sequence is transmitted to channel $h_{u}(\tau)$.

\subsection{Channel}

In order to clearly show the effectiveness of the proposed DS-CDMA, we assume time-invariant two-path channels in most of this study. The relative delay time $\tau_{u}$ [s] of the twopath channel for the $u$ th user is assumed to be $\tau_{u}=\tau_{u 2}-\tau_{u 1}$, where $\tau_{u 1}$ and $\tau_{u 2}\left(\tau_{u 1}<\tau_{u 2}\right)$ are the time delays for the first and second paths of the channel for the $u$ th user, respectively. Figure 3 illustrates the signals received through a two-path channel, where the length $L$ of the spreading sequence is assumed to be $L=7$ (and $L+\alpha$ is the size of the COF, as described below).

\subsection{Receiver}

The receiver is composed of the COF. The COF has $L+$ $\alpha(0 \leq \alpha \leq L)$ tap weights, $c_{u, 1}, c_{u, 2}, \ldots, c_{u, L+\alpha}$, and the tap weights are updated by an adaptive algorithm, as shown in Fig. 2. Here, we adopt a normalized least-mean-square (N-LMS) algorithm [6], and the time interval of updates is assumed to be $T_{s}$. For simplicity, we assume that the receiver for the $u$ th user is synchronized to the first path (i.e., initial synchronization to timing $\tau_{u 1}$ is perfect).

Received signal samples $r(n)$ can be obtained by sampling the signal at time $t=n T_{c}(n=1,2, \ldots)$ through a chip-matched filter, as shown in Fig. 2. We represent the received signal samples $r(n)$ being retained in the COF at $t=(k L+\alpha) T_{c}(k=1,2, \ldots)$ by a vector form $\mathbf{r}(k)$ as

$$
\begin{aligned}
\mathbf{r}(k)= & {[r((k-1) L+1), r((k-1) L+2),} \\
& \ldots, r(k L), r(k L+1), \\
& r(k L+2), \ldots, r(k L+\alpha)]^{T},
\end{aligned}
$$

where the superscript ${ }^{T}$ denotes the transpose of the vector.

We also represent the tap weights of the COF for the $u$ th user at $t=(k L+\alpha) T_{c}$ by a vector $\mathbf{c}_{u}(k)$ as

$$
\begin{array}{r}
\mathbf{c}_{u}(k)=\left[c_{u, 1}(k), c_{u, 2}(k), \ldots, c_{u, L}(k), c_{u, L+1}(k),\right. \\
\left.c_{u, L+2}(k), \ldots, c_{u, L+\alpha}(k)\right]^{T} .
\end{array}
$$

The tap weight vector $\mathbf{c}_{u}(k)$ is first updated by the $\mathrm{N}$ LMS algorithm [6] as follows:

$$
\begin{aligned}
& \tilde{\mathbf{c}}_{u}(k+1) \\
& \quad=\mathbf{c}_{u}(k)+\frac{\mu}{\|\mathbf{r}(k)\|^{2}} \mathbf{r}(k)\left[d_{u}(k)-\mathbf{r}(k)^{T} \mathbf{c}_{u}(k)\right],
\end{aligned}
$$

where $\mu$ is the step-size parameter, $\|\cdot\|$ denotes the norm of the enclosed vector, and $d_{u}(k)$ is the reference ( $k$ th data bit) of the $u$ th user. We assume a decision-directed reference $d_{u}(k)=\operatorname{sgn}\left[\mathbf{r}(k)^{T} \mathbf{c}_{u}(k)\right](\operatorname{sgn}[x]=\{+1(x>0),-1(x<0)\})$ at $t=(k L+\alpha) T_{c}^{\dagger}$.

Then, shortly afterwards, the magnitude of the tap weight vector is adjusted by

$$
\mathbf{c}_{u}(k+1)=\tilde{\mathbf{c}}_{u}(k+1) /\left\|\tilde{\mathbf{c}}_{u}(k+1)\right\| .
$$

This means that the tap weight vector $\mathbf{c}_{u}(k)$ is updated subject to the unit norm in the COF. Based on (3) and (4), the updates of $\mathbf{c}_{u}(k)$ are performed at $t=(k L+\alpha) T_{c}$ (i.e., at every time instant for the data decision).

\subsection{Proposed FCSS/DS-CDMA}

\subsubsection{Feedback-Controlled Spreading Sequence (FCSS)}

In the FCSS/DS-CDMA, the receiver for the $u$ th user returns a part of $\mathbf{c}_{u}(k)$, that is, $c_{u, 1}(k), c_{u, 2}(k), \ldots, c_{u, L}(k)$, to the transmitter every $T_{f}=N T_{s}[\mathrm{~s}]$ ( $N$ is a positive integer) specified for the feedback $M_{u}$ times, as illustrated in Fig. 2, and then, the transmitter uses the sequence $c_{u, 1}(k), c_{u, 2}(k), \ldots, c_{u, L}(k)$ as its spreading sequence. Therefore, the spreading sequence for the $u$ th user at $t=m T_{f}+\alpha T_{c}=m N T_{s}+\alpha T_{c}$

${ }^{\dagger}$ As for producing the analog spreading sequences [9], the reference $d(k)$ must be $d(k)=0$. In addition, the COF size must be identical to the length $L$ of spreading sequences. 


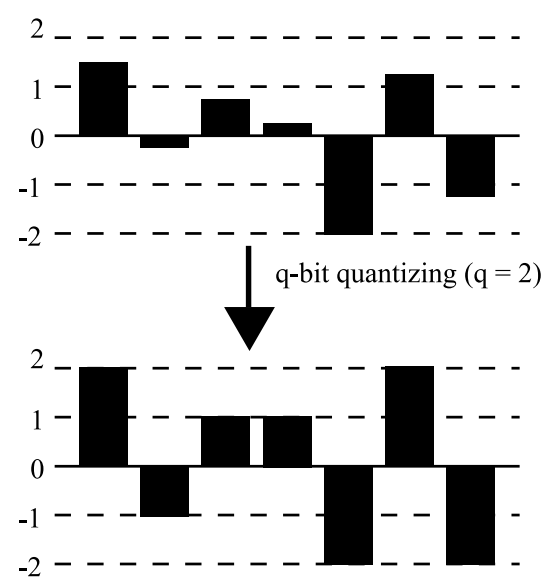

Fig. 4 Example of quantized FCSS $(q=2, L=7)$.

( $m=0,1, \ldots, M_{u}$ ) can be represented by a vector form $\mathbf{p}_{u}(m)$ as

$$
\begin{gathered}
\mathbf{p}_{u}(m)\left(\triangleq\left[p_{u, 1}(m), p_{u, 2}(m), \ldots, p_{u, L}(m)\right]^{T}\right) \\
=\left[c_{u, 1}(m N), c_{u, 2}(m N), \ldots, c_{u, L}(m N)\right]^{T} .
\end{gathered}
$$

In this paper, $\mathbf{p}_{u}(m)$ is referred to as the feedback-controlled spreading sequence (FCSS). Although all $U$ users can choose distinct values of the feedback interval, we assume, for simplicity, that all users choose an identical value of $T_{f}$.

The feedback functions to train the DS-CDMA. The COF produces the tap weights that are the minimum meansquared error (MMSE) solution to the reference $d_{u}(k)$ resulting in minimum ISI and MAI for the present received signals. The feedback changes the transmitted signal, thereby forming another received signal. According to the different received signal, the COF can produce different tap weights. In this paper, we show that the feedback forms better received signals efficiently without any explicit knowledge of the channel and information of simultaneously communicating other users in the asynchronous DS-CDMA over timedispersive channels. Note that for the respective receivers, only sending the feedback part of own COF tap weights to the corresponding transmitter is required. Therefore, the proposed FCSS/DS-CDMA is applicable to the decentralized multiple access.

\subsubsection{Quantization}

Since the tap weights take analog values, the receiver requires quantization for the elements of the FCSS in order to send them to the transmitter as binary data bits.

As shown in Fig. 4, we assume uniform quantization that is confined to the range $\left[-\left|c_{u, l}(m N)\right|_{\max },+\left|c_{u, l}(m N)\right|_{\max }\right]$, where $\left|c_{u, l}(m N)\right|_{\text {max }}$ denotes the maximum absolute value of the elements $c_{u, l}(m N)(l \in[1, L])$ of the feedback part of the tap weights at $t=m N T_{s}+\alpha T_{c}$ in the COF.

Suppose that $q$ denotes the number of quantizing bits per chip and $[\cdot]_{q}$ represents a vector, each element of which is quantized with $q$ bits. Hence, the transmitter uses a quantized version $\left[\mathbf{p}_{u}(m)\right]_{q}$ of the $\operatorname{FCSS} \mathbf{p}_{u}(m)$ when the influence of quantization is considered. $q L$ bits are required to send one FCSS to the transmitter, and $q L M_{u}$ bits are required in total, because the receiver repeats the feedback $M_{u}$ times with the interval $T_{f}$. In this paper, we assume that the feedback is perfectly achieved (i.e., no transmission error is assumed for the feedback).

\section{Simulation Results}

In this section, we show several characteristics of the FCSS/DS-CDMA in the presence of an additive white Gaussian noise (AWGN) with double-sided power spectral density $N_{0} / 2[\mathrm{~W} / \mathrm{Hz}]$. We assume that the COF uses the decision-directed reference $d_{u}(k)=\operatorname{sgn}\left[\mathbf{r}(k)^{T} \mathbf{c}_{u}(k)\right]$. For the COF of the $u$ th user, the initial values $\mathbf{c}_{u}(0)$ of the tap weights of size $L+\alpha(0 \leq \alpha \leq L)$ are set to a sequence that consists of the corresponding initial spreading sequence $\mathbf{p}_{u}(0)$ and its serial connection to make the length of the sequence suited to the size $L+\alpha$ of the tap weights, that is,

$$
\begin{array}{r}
\mathbf{c}_{u}(0)=\left[p_{u, 1}(0), p_{u, 2}(0), \ldots, p_{u, L}(0), p_{u, 1}(0),\right. \\
\left.p_{u, 2}(0), \ldots, p_{u, \alpha}(0)\right]^{T} .
\end{array}
$$

Another choice for $\mathbf{c}_{u}(0)$ will be

$$
\mathbf{c}_{u}(0)=[p_{u, 1}(0), p_{u, 2}(0), \ldots, p_{u, L}(0), \underbrace{0,0, \ldots, 0}_{\alpha \text { zeros }}]^{T} .
$$

In this paper, we employ (6) as an example.

\subsection{Single-User Performance}

First, we investigate several characteristics of the FCSS/DSCDMA for $U=1$ (single-user case) over a two-path equalgain channel. A maximum length sequence (M-sequence) of length $L=7$ is assumed to be initially employed as the spreading sequence $\mathbf{p}_{1}(0)=[-,-,+,-,+,+,-]^{T}$ for the user $(u=1)$ in the FCSS/DS-CDMA. This is also employed in the conventional DS-CDMA. The relative delay $\tau_{u}$ of the channel is assumed to be $\tau_{u}=4 T_{c}$. The received energy per bit for the first path is represented by $E_{b 1}$, and for the second path, $E_{b 2}$, and they are assumed to be identical $\left(E_{b 1}=E_{b 2}\right)$.

\subsubsection{Converged Weights and FCSS}

Figure 5 shows an example of converged tap weights $\mathbf{c}_{u}\left(10^{7}\right)$ of the COF with $L+\alpha=2 L(=14)$, observed at $t=$ $10^{7} T_{s}+7 T_{c}$, for $u=1$ and $U=1$, provided that the feedback interval $T_{f}=10^{5} T_{s}\left(N=10^{5}\right)$. In order to ensure the convergence, the feedback times $M_{1}$ was chosen to be large $\left(M_{1}=100\right)$. Note that the feedback part of $\mathbf{c}_{1}\left(10^{7}\right)$, that is, $c_{1,1}\left(10^{7}\right), c_{1,2}\left(10^{7}\right), \ldots, c_{1, L}\left(10^{7}\right)$, corresponds to the converged FCSS $\mathbf{p}_{1}(100)$ as denoted in Fig. 5, except for a trivial difference in amplitude.

It is important to examine the property of crosscorrelation between the received signal vector and the converged COF weight vector to clarify the performance of the FCSS/DS-CDMA. Usually, the odd cross-correlation, rather 


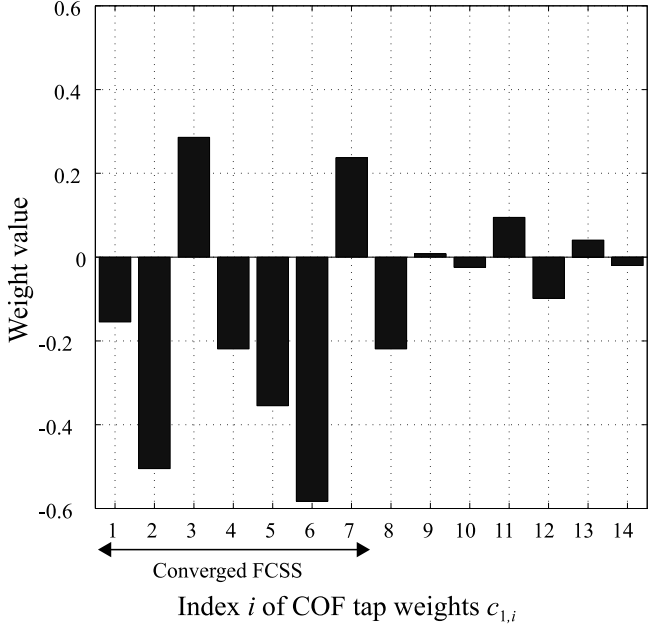

Fig.5 Converged COF weights and FCSS ( $U=1$, no quantization, $T_{f}=$ $\left.10^{5} T_{s}\right)$

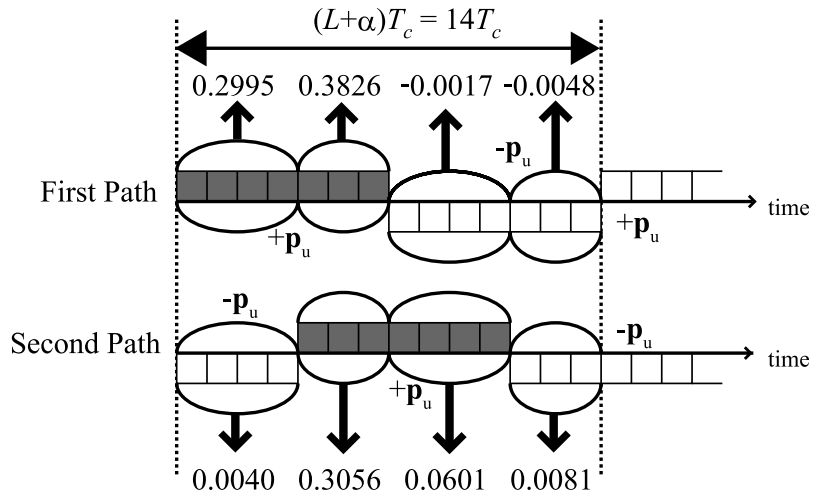

Fig. 6 Partial closs-correlation values between the converged signals and COF tap weights ( $U=1$, no quantization, $T_{f}=10^{5} T_{s}$ ).

than the even cross-correlation, is laborious to make into a small value in DS-CDMA [13], particularly for spreading sequences of short length.

Figure 6 illustrates the received signals through the first and second paths for the odd cross-correlation case. The numerical values given in Fig. 6 indicate partial crosscorrelation values between the COF weights $\left(\left\|\mathbf{c}_{1}\left(10^{7}\right)\right\|^{2}=\right.$ 1) shown in Fig. 5 and the received signals $\left(\left\| \pm \mathbf{p}_{1}(100)\right\|^{2}=\right.$ $1 / 2$ ) illustrated in Fig. 6 . The +1 , binary bit colored gray in Fig. 6, are being detected at this time instant. The sum of all eight numerical values corresponds to the $\mathrm{COF}$ output that is used to decide whether data are +1 or -1 .

Note that the sum of ISI components caused by the adjacent data approximately reduces to zero (i.e., $-0.0017-$ $0.0048+0.0040+0.0081=0.0056$ ), whereas the sum of the desired components becomes one (i.e., $0.2995+0.3826+$ $0.3056+0.0601=1.0478)$. This means that the desired data can be detected without the influence of the ISI in the FCSS/DS-CDMA.

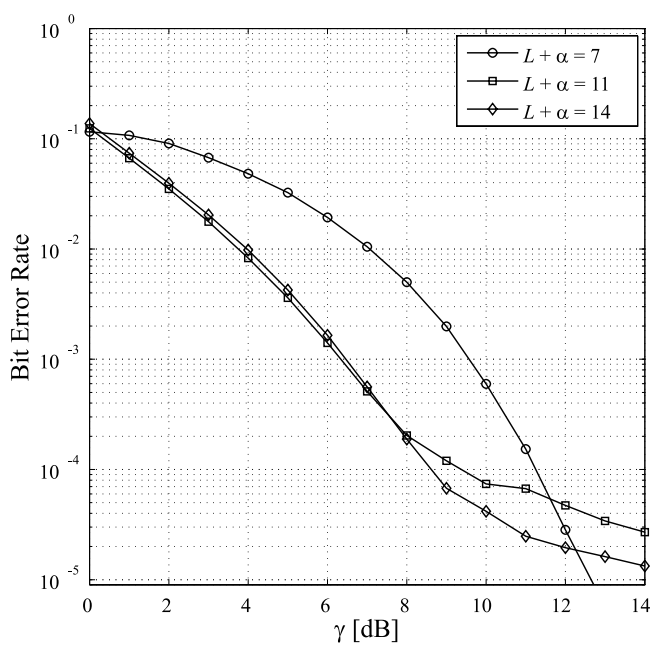

Fig. 7 BER performance vs. the number of COF tap weights $(U=1$, no quantization, early period, $T_{f}=T_{s}$ ).

\subsubsection{BER Performance}

Since the spreading sequence varies with time in the FCSS/DS-CDMA, BER performance also varies with time. Therefore, for the proposed FCSS/DS-CDMA, we evaluate the BER characteristics not only in the early period but also in the steady period. In this paper, the early period is defined as the period until we receive the data stream of $10^{7}$ bits, and the steady period is defined as the period after receiving $10^{7}$ bits of data.

Figure 7 shows BER performance of the FCSS/DSCDMA in the early period as a function of $\gamma\left(\triangleq\left(E_{b 1}+\right.\right.$ $\left.\left.E_{b 2}\right) / N_{0}\right)$ in $\mathrm{dB}$. The number of COF tap weights, $L+\alpha$, was chosen to be $L+\alpha=7,11$ and 14 (i.e., $\alpha=0,4$ and 7). Since the main purpose of Fig. 7 is to determine a favorable number of COF tap weights, the feedback interval $T_{f}$ for updating the FCSS was chosen to be $T_{f}=T_{s}$ for simplicity. As can be seen in Fig. 7, the cases $L+\alpha=11$ and 14 show almost identical performance when $\gamma \leq 8 \mathrm{~dB}$. This is because $L+\alpha \geq L+\tau_{u}(=11)$. Usually, the relative delay $\tau_{u}$ is unknown, $L+\alpha=2 L(=14)$ will be a better choice as the size of the COF tap weights.

Next we investigate the relationship between the BER performance and the feedback time interval $T_{f}$. Figure 8 shows the BER performance of the FCSS/DS-CDMA in the early period. Simulations were performed with $T_{f}=$ $10^{0} T_{s}, 10^{1} T_{s}, \ldots$, and $10^{6} T_{s}$. Note that $T_{f}$ shorter than $10^{5} T_{s}$ is sufficient to achieve good BER performance in the early period that corresponds to a $10^{7}$-bit duration.

Figure 9 shows BER characteristics of the FCSS/DSCDMA in comparison with the conventional DS-CDMA with/without RAKE combining (two-finger equal-gain combining) as a function of $\gamma$ in $\mathrm{dB}$. Since the M-sequence of length $L=7$ has a large odd cross-correlation, the performance of the conventional DS-CDMA is greatly influenced by the ISI due to the multipath even though RAKE combin- 


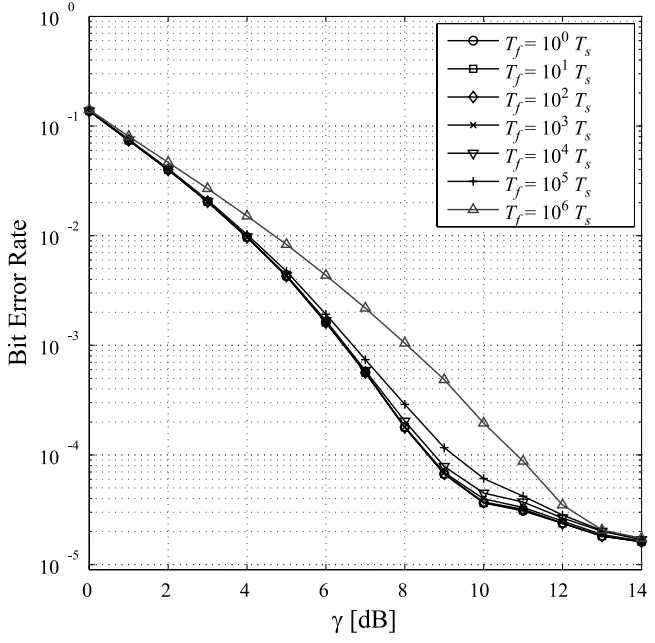

Fig. 8 BER performance vs. feedback time intervals $(U=1$, no quantization, early period, $L+\alpha=14$ ).

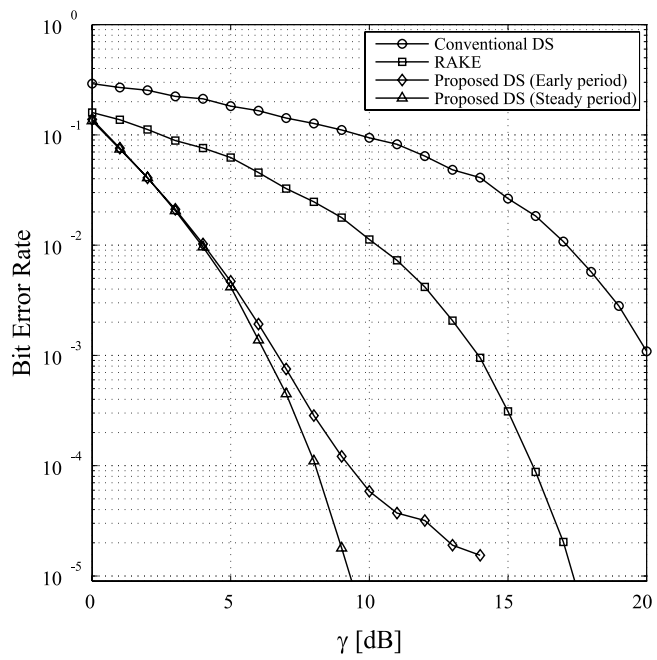

Fig.9 BER performance of the FCSS/DS-CDMA (no quantization, $L+$ $\alpha=14, T_{f}=10^{5} T_{s}$ ) in comparison with the conventional DS-CDMA with/without RAKE combining (2-finger equal-gain RAKE) for $U=1$.

ing is adopted.

Note that BER characteristics for the conventional DSCDMA largely depend on the correlation function of the spreading sequence of choice, and a worse-case performance is shown in Fig. 9. The results indicate that the FCSS/DS-CDMA greatly improves the performance of the conventional DS-CDMA in both the early and the steady period, even though the initially chosen spreading sequence has a poor correlation function.

Next, we show the characteristics of the BER performance vs. the feedback times $M_{1}$ in Fig. 10 . Note that the BER characteristic for no feedback case $\left(M_{1}=0\right)$ corresponds to the case of the conventional DS-CDMA with a COF receiver.

As can be seen in Fig. 10, a considerable improvement in the BER can be obtained with the first feedback $\left(M_{1}=1\right)$. In addition, it is clear that two or three repetitions $\left(M_{1}=2\right.$

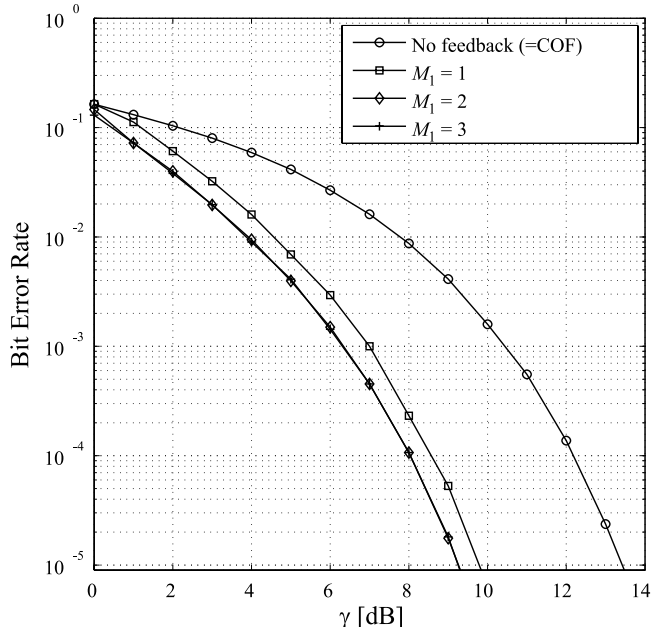

Fig. 10 BER performance vs. feedback times $M_{1}(U=1$, no quantization, steady period, $L+\alpha=14, T_{f}=10^{5} T_{s}$ ).

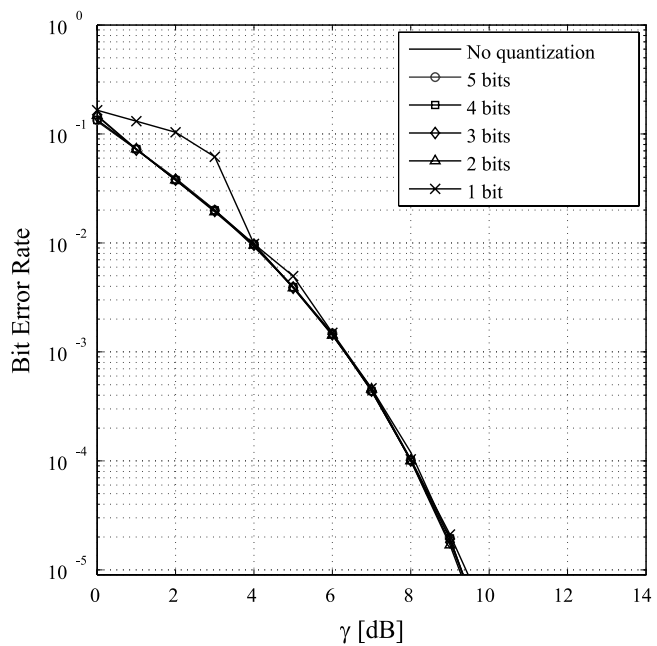

Fig. 11 BER performance vs. quantizating bits per chip $q(U=1$, steady period, $L+\alpha=14, T_{f}=10^{5} T_{s}, M_{1}=3$ ).

or 3) of feedback are sufficient in the BER for the single user over the two-path channel we assumed.

Figure 11 shows the relationship between the BER performance and the number of quantizing bits per chip $q$. The feedback times $M_{1}$ was chosen to be $M_{1}=3$. Interestingly, almost no influence of the number of quantizing bits occurs in the BER. This is because the spreading sequence of length $L=7$ has a sufficiently higher degree of freedom in order to suppress the ISI over the two-path channel.

\subsection{Asynchronous Multiple Access Performance}

Next, we evaluate the multiple access performance for three users $(U=3)$. The initial spreading sequences for the three users in the FCSS/DS-CDMA are chosen to be $\mathbf{p}_{1}(0)=[-,-,+,-,+,+,-]^{T}, \mathbf{p}_{2}(0)=[+,+,+,+,+,+,+]^{T}$, and $\mathbf{p}_{3}(0)=[+,+,-,-,+,+,-]^{T}$, respectively. Note that there is a probability that the tap weights of $\mathrm{COF}$ with 
decision-directed algorithm converge to the weights that recover the data of other users for case of the multiple access. In order to avoid such a worst-case scenario, known data bits that are prescribed for the COF training can be adopted as the reference in realistic applications.

We assume that the first user starts the transmission at
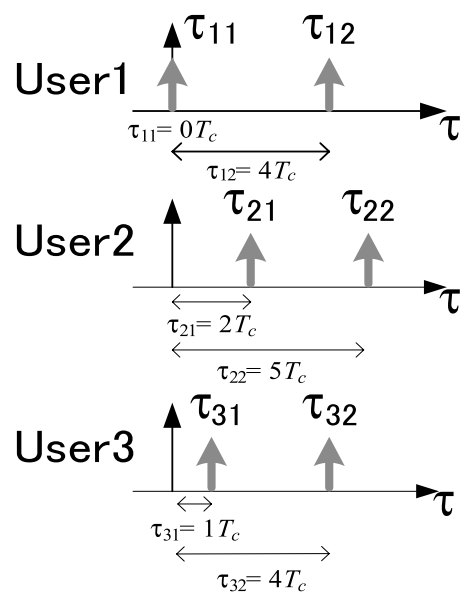

Fig. 12 Channel impulse responses for users 1, 2, and 3. $t=0$, the second user starts at $t=T_{f}[\mathrm{~s}]$ and the third user starts at $t=2 T_{f}[\mathrm{~s}]$, and $M_{1}=M_{2}+1=M_{3}+2$ for the feedback times $M_{u}$. Therefore, all users finish the feedback at $t=M_{3} T_{f}+\alpha T_{c}$ at the same time.

Figure 12 shows the channel impulse responses for

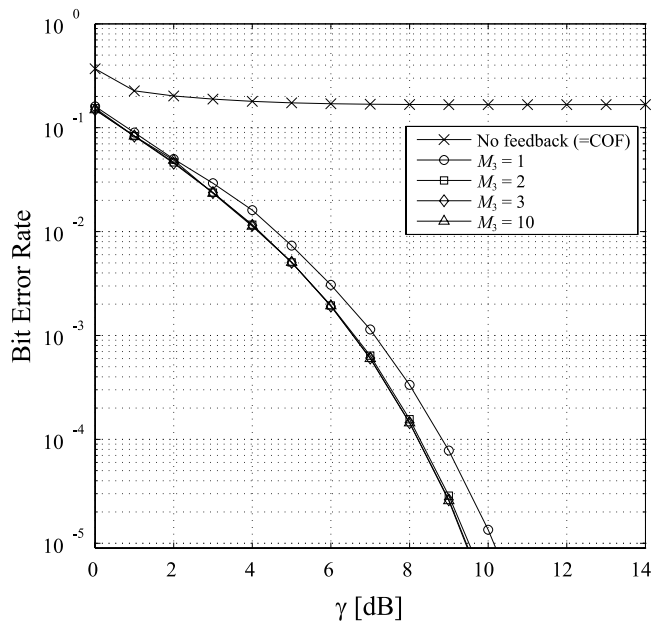

Fig. 13 BER performance vs. feedback times $M_{3}$ for $U=3$ (no quantization, steady period, $\left.L+\alpha=14, T_{f}=10^{5} T_{s}, M_{1}=M_{2}+1=M_{3}+2\right)$.

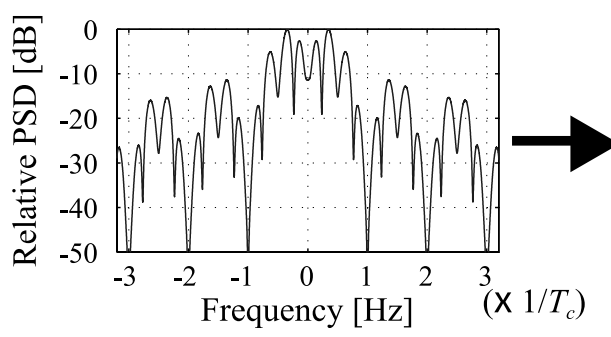

(a)

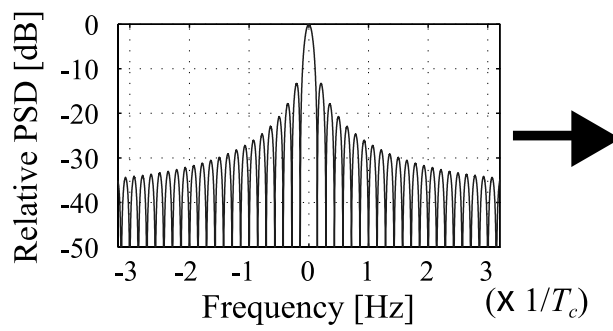

(c)

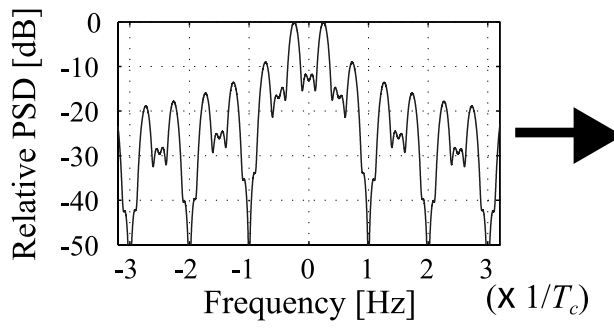

(e)

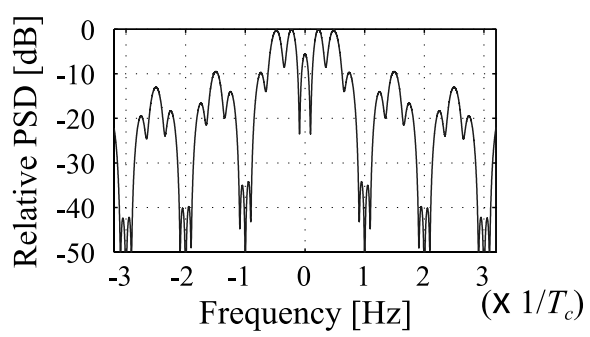

(b)

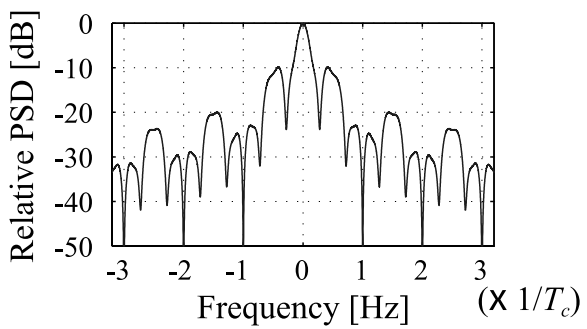

(d)

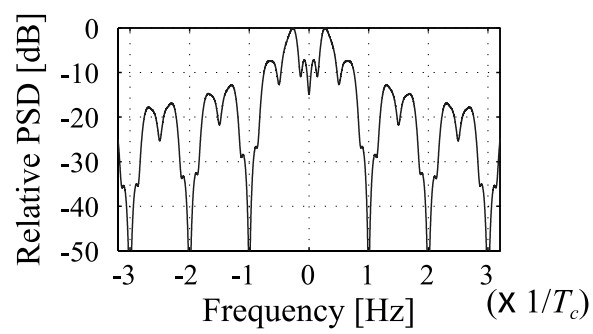

(f)

Fig. 14 Relative power spectral density (PSD) of the FCSS/DS signals for respective users transmitted in the steady period for the scenario of Fig. $13\left(M_{3}=3\right)((a)$ and (b): Initial sequence and FCSS for user 1; (c) and (d): for user 2; (e) and (f): for user 3). 


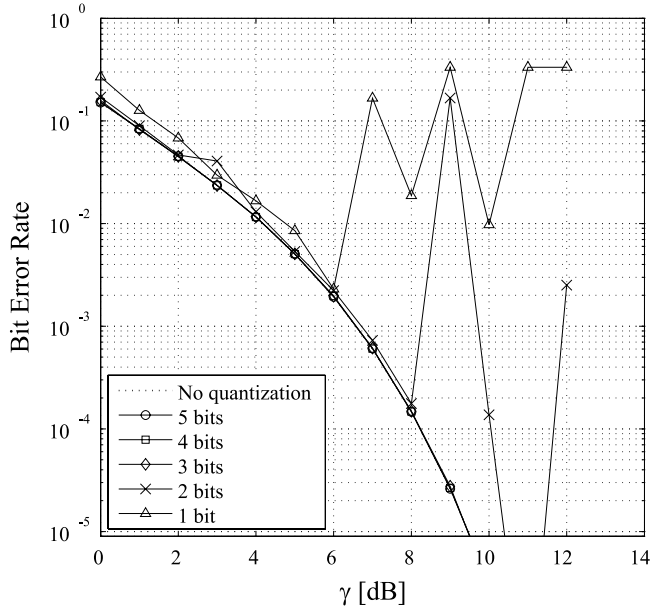

Fig. 15 BER performance vs. quantizing bits per chip $q$ for $U=3$ (steady period, $L+\alpha=14, T_{f}=10^{5} T_{s}, M_{1}=M_{2}+1=M_{3}+2=5$ ).

three users (i.e., user 1, user 2, and user 3). The excess delays of the channels are assumed to be $\tau_{11}=0$ and $\tau_{12}=4 T_{c}$ for user $1, \tau_{21}=2 T_{c}$ and $\tau_{22}=5 T_{c}$ for user 2 , and $\tau_{31}=1 T_{c}$ and $\tau_{32}=4 T_{c}$ for user 3 . The received energy per bit for the first path is represented by $E_{b 1}$, and for the second path, by $E_{b 2}$, and they are assumed to be identical $\left(E_{b 1}=E_{b 2}\right)$ for all users.

Figure 13 shows the relationship between the BER performance and the feedback times $M_{3}$. The values of the BER are the average for the three users.

As can be observed in Fig. 13, the FCSS/DS-CDMA ( $U=3$ ) achieves almost identical BER performance to that of the single-user case (see Fig. 10), whereas the multiple access degrades the BER performance of the $\mathrm{COF}$ receiver $\left(M_{1}=M_{2}=M_{3}=0\right)$. It can be seen from Fig. 13 that two or three repetitions $\left(M_{3}=2\right.$ or 3$)$ of feedback for the finally added user (i.e., the third user) are sufficient in the BER for $U=3$ over the two-path channel we assumed. The power spectra of the signals transmitted by the respective users in the steady period for the scenario of Fig. 13 are shown in Fig. 14 for $M_{3}=3$.

Figure 15 shows the relationship between the BER performance and the number of quantization bits per chip $q$ for $U=3$ and $M_{3}=3$ (i.e., $M_{1}=M_{2}+1=M_{3}+2=5$ ). In Fig. 15, it can be seen that a great number of quantizing bits is required, in comparison with the single-user case (see Fig. 11), to gain a better performance of BER in the multiple access environment. For the case of Fig. 15, 3- or 4-bit quantization is sufficiently good in the BER.

\subsection{Powerline Communication Performance}

In order to test the performance of the FCSS/DS-CDMA over a channel that causes a severe distortion to the transmitted signals, we adopt a powerline communication (PLC) channel of VVF (vinyl insulation, vinyl sheath, flat) cables commonly used for powerlines in Japan. Figure 16 shows a model of a powerline [14].

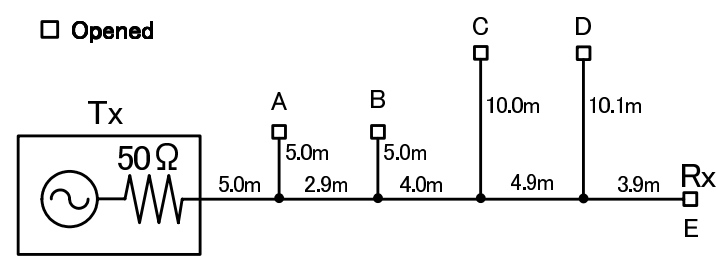

Fig. 16 Model of powerline channel.

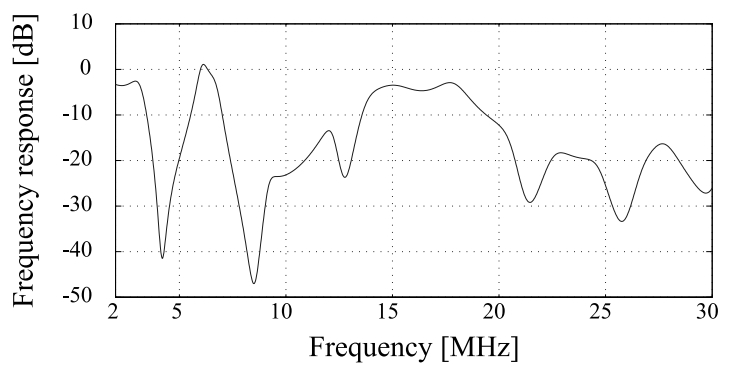

(a)

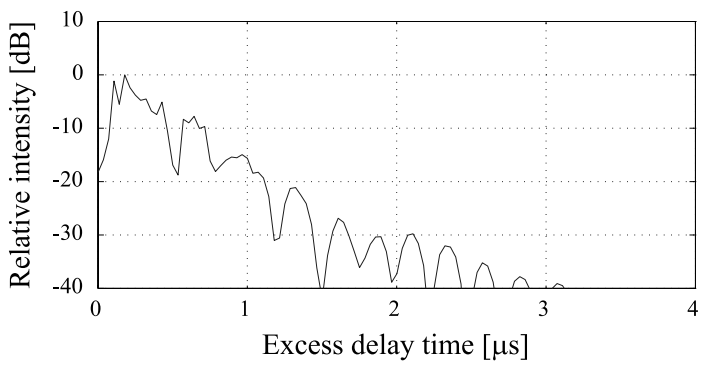

(b)

Fig. 17 (a) Transfer function of the powerline channel, and (b) corresponding impulse response.

The VVF cable consists of two wires of $\phi 1.6 \mathrm{~mm}$, and branch cables A, B, C, D and E are connected as shown in Fig. 16. The receiver $R_{x}$ is set at the terminal of branch cable E.

Figure 17(a) shows the frequency response [14] of the PLC channel where all terminals of the branch cables are assumed to be opened, and the corresponding impulse response is shown in Fig. 17(b).

\subsubsection{BER Performace}

For the PLC channel, the center frequency and chip rate $1 / T_{c}$ are assumed to be $16 \mathrm{MHz}$ and $14 \mathrm{MHz}$, respectively. Because of $T_{s}=L T_{c}$ and $L=7$, data rate $1 / T_{s}$ becomes $2 \mathrm{Mbps}$. In order to avoid scattered plots of BER and to confirm a converged performance in the steady period, we assume the feedback with $T_{f}=10^{4} T_{s}$ and $M_{1}=10^{3}$ for the simulations. As for the realistic applications of the PLC, the peak power of the spectrum must be kept lower than that of the maximum allowable power per frequency; however, we forgive unconsidered simulations for employing the PLC channel for the purpose of only testing the proposed FCSS/DS-CDMA.

Figure 18 shows BER characteristics for $U=1$ in both 


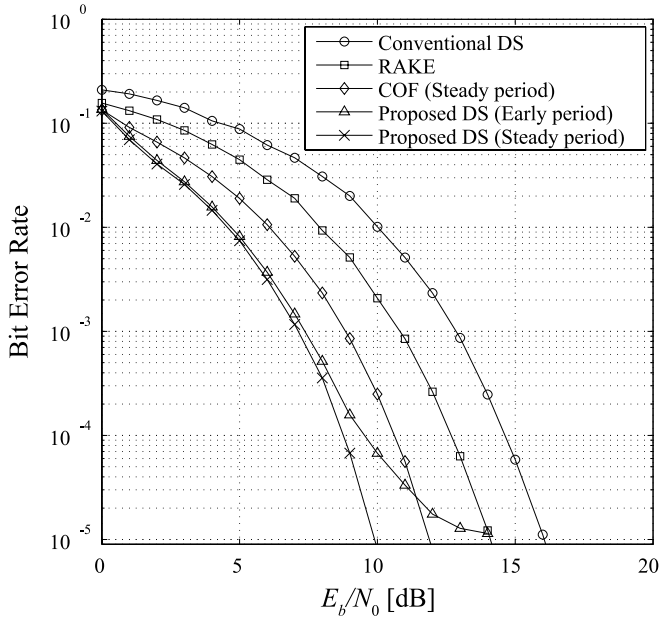

Fig. 18 BER performances of the FCSS/DS-CDMA (No quantization, $\left.L+\alpha=14, T_{f}=10^{4} T_{s}, M_{1}=10^{3}\right)$ for $U=1$ in comparison with the conventional DS-CDMA with/without RAKE combining (2-finger equalgain RAKE) and with COF.

the early and the steady period as a function of $E_{b} / N_{0}$ in $\mathrm{dB}$ where $E_{b}$ denotes the energy per bit at the receiver $\mathrm{R}_{\mathrm{x}}$. Note that the BER characteristic for the conventional DSCDMA corresponds to the initial characteristic for the proposed FCSS/DS-CDMA and the COF corresponds to the case for no feedback. As can be seen in Fig. 18, the BER performance can be greatly improved through the feedback process of the proposed DS-CDMA even for the channels that cause severe distortions.

\section{Conclusion}

In this paper, a novel FCSS/DS-CDMA was proposed and asynchronous DS-CDMA performance was evaluated by simulation in terms of the BER over time-invariant two-path and powerline channels.

As a result, it was shown that the proposed FCSS/DSCDMA greatly suppresses both ISI and MAI, and that a significant improvement in BER performance can be obtained over time-dispersive channels.

\section{Acknowledgment}

This work was supported in part by a Grant-in-Aid for Young Scientists (B) (No. 19760271) from the Ministry of Education, Culture, Sports, Science and Technology (MEXT) in Japan.

\section{References}

[1] J.G. Proakis, Digital Communications, 3rd ed., McGraw-Hill, New York, 1995

[2] W.C. Jakes, Microwave Mobile Communications, IEEE Press, Piscataway, NJ, 1974

[3] T.S. Rappaport, Wireless Communications, 2nd ed., Prentice Hall, Englewood Cliffs, NJ, 2002.

[4] G. Marubayashi, M. Nakagawa, and R. Kohno, Spread Spectrum Communications and Its Applications, IEICE, Tokyo, 1998.
[5] R. Esmailzadeh and M. Nakagawa, "Pre-RAKE diversity combination for direct-sequence spread spectrum mobile communications systems," IEICE Trans. Commun., vol.E76-B, no.8, pp.1008-1015, Aug. 1993.

[6] S. Haykin, Adaptive Filter Theory, 3rd ed., Prentice Hall, Englewood Cliffs, NJ, 1996.

[7] S. Yoshida, A. Ushirokawa, S. Yanagi, and Y. Furuya, "DS/CDMA adaptive interference canceller on differential detection for fast fading channel," IEICE Trans. Commun. (Japanese Edition), vol.J77B-II, no.11, pp.618-627, Nov. 1994.

[8] U. Madhow and M.L. Honig, "MMSE interference suppression for direct-sequence spread-spectrum CDMA," IEEE Trans. Commun., vol.42, pp.3178-3188, Dec. 1994.

[9] S. Hamada, M. Hamamura, H. Suzuki, and S. Tachikawa, "A proposed DS/CDMA system using analog PN sequences produced by adaptive filters," IEICE Trans. Fundamentals, vol.E81-A, no.11, pp.2261-2268, Nov. 1998.

[10] T. Miyatake, K. Yamakawa, M. Hamamura, and S. Tachikawa, "A DS/SS system with feedback loop for spreading sequence," 2003 Shikoku Section Joint Convention Record of the Institutes of Electrical and Related Engineers, p.200, Oct. 2003.

[11] T. Miyatake, M. Hamamura, and S. Tachikawa, "Performance of DS/SS system using feedback controlled spreading sequence over a multipath channel," Proc. ISITA2004, pp.567-571, Parma, Italy, Oct. 2004.

[12] T. Miyatake, M. Hamamura, and S. Tachikawa, "Combining characteristics of DS/SS system using feedback controlled spreading sequence for time-dispersive channels," IEICE Technical Report, WBS2004-45, Dec. 2004.

[13] M.B. Pursley, "Performance evaluation for phase-coded spreadspectrum multiple-access communication-Part I: System analysis," IEEE Trans. Commun., vol.COM-25, no.8, pp.795-799, Aug. 1977.

[14] S. Tsuzuki, T. Takamatsu, H. Nishio, and Y. Yamada, "An estimation method of the transfer function of indoor power-line channels for Japanese houses," Proc. ISPLC2002, pp.55-59, March 2002.

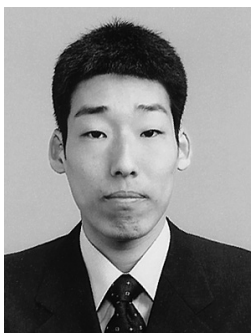

Teruhiko Miyatake received his B.E and M.E. degrees in Information Systems Engineering from Kochi University of Technology, Kochi, Japan in 2004 and 2006, respectively. His research interests lie in the area of adaptive signal processing.

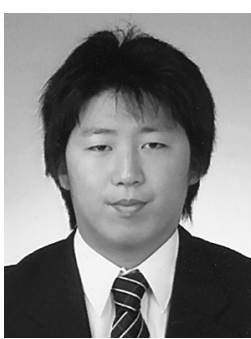

Kazuki Chiba is currently working toward the B.E. degree in Information System Engineering at Kochi University of Techonology, Kochi, Japan. His research interests lie in the area of feedback-based communication systems. 


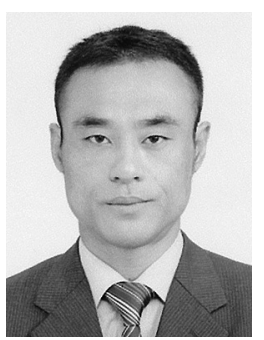

Masanori Hamamura received his B.S., M.S. and Ph.D. degrees in electrical engineering from Nagaoka University of Technology, Nagaoka, Japan, in 1993, 1995 and 1998, respectively. From 1998 to 2000, he was a Research Fellow of the Japan Society for the Promotion of Science. Since 2000, he has been with the Department of Information Systems Engineering at Kochi University of Technology, Kochi, Japan, where he is now an Associate Professor. His current research interests are in the areas of spread spectrum systems, mobile communications and signal processing. Dr. Hamamura is a member of IEEE and SITA of Japan.

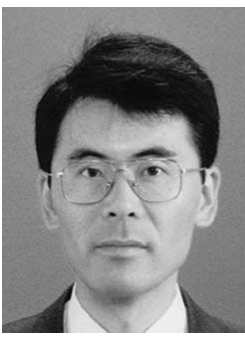

SITA of Japan.
Shin'ichi Tachikawa received the B.S. M.S. and Dr. degrees in electrical engineering from Nagaoka University of Technology, Nagaoka, Japan, in 1980, 1982 and 1991, respectively. Since 1982, he has been a faculty member of Engineering at Nagaoka University of Technology, where he is now an Associate Professor. His current research interests lie in the areas of spread spectrum communication system, wide band system, coding theory and signal processing. He is a member of IEEE and 